PROCEEDINGS OF THE

AMERICAN MATHEMATICAL SOCIETY

Volume 127, Number 10, Pages 2821-2827

S 0002-9939(99)04976-X

Article electronically published on May 4, 1999

\title{
SOME LIE SUPERALGEBRAS ASSOCIATED TO THE WEYL ALGEBRAS
}

\author{
IAN M. MUSSON
}

(Communicated by Ken Goodearl)

\begin{abstract}
Let $\mathfrak{g}$ be the Lie superalgebra $\operatorname{osp}(1,2 r)$. We show that there is a surjective homomorphism from $U(\mathfrak{g})$ to the $r^{t h}$ Weyl algebra $A_{r}$, and we use this to construct an analog of the Joseph ideal. We also obtain a decomposition of the adjoint representation of $\mathfrak{g}$ on $A_{r}$ and use this to show that if $A_{r}$ is made into a Lie superalgebra using its natural $\mathbb{Z}_{2}$-grading, then $A_{r}=k \oplus\left[A_{r}, A_{r}\right]$. In addition, we show that if $\left[A_{r}, A_{r}\right]$ and $\left[A_{s}, A_{s}\right]$ are isomorphic as Lie superalgebras, then $r=s$. This answers a question of $\mathrm{S}$. Montgomery.
\end{abstract}

We work throughout over an algebraically closed field $k$ of characteristic zero. If $\mathfrak{g}$ is a simple Lie algebra different from $s \ell(n)$, Joseph shows in [J2], that there is a unique completely prime ideal, $J_{0}$ whose associated variety is the closure of the minimal nilpotent orbit in $\mathfrak{g} *$. When $\mathfrak{g}$ is the symplectic algebra $\mathfrak{g}=s p(2 r)$, this ideal may be constructed as follows. It is well known that the symmetric elements of degree two in the $r^{t h}$ Weyl algebra $A_{r}$ form a Lie algebra isomorphic to $s p(2 r)$ [D, Lemma 4.6.9]. Hence there is an algebra map $\phi: U(\mathfrak{g}) \longrightarrow A_{r}$ whose kernel is clearly completely prime and primitive. Since the image of $\phi$ has Gel'fand Kirillov dimension $2 r$, and this is the dimension of the minimal nilpotent orbit in $\mathfrak{g} *$ by [CM, Lemma 4.3.5], we have $\operatorname{ker} \phi=J_{0}$.

Now if $\mathfrak{g}$ is a classical simple Lie superalgebra, and $U(\mathfrak{g})$ contains a completely prime primitive ideal different from the augmentation ideal, then $\mathfrak{g}$ is isomorphic to an orthosymplectic algebra $\operatorname{osp}(1,2 r)$ (Lemma 1$)$. We observe that if $\mathfrak{g}=\operatorname{osp}(1,2 r)$, then there is a surjective homomorphism $U(\mathfrak{g}) \longrightarrow A_{r}$ whose kernel $J$ satisfies $J \cap U\left(\mathfrak{g}_{0}\right)=J_{0}$. The existence of this homomorphism has previously been shown in the Physics literature; see, for example, [F, pages 55 and 170]. It follows that $\mathfrak{g}$ acts via the adjoint representation on $A_{r}$, and we determine the decomposition of this representation explicitly.

This turns out to be a useful setting in which to study the Lie structure of certain associative algebras. A result of Herstein [He] states that if $A$ is a simple algebra with center $Z$, then $[A, A] /[A, A] \cap Z$ is a simple Lie algebra, unless $[A: Z]=4$, and $Z$ has characteristic two. Additional results have been obtained for various generalized Lie structures in $[\mathrm{BFM}]$ and $[\mathrm{Mo}]$.

Received by the editors February 7, 1997 and, in revised form, December 9, 1997.

1991 Mathematics Subject Classification. Primary 17B35; Secondary 16W10.

Key words and phrases. Lie superalgebras, Weyl algebras, Joseph ideal.

This research was partially supported by NSF grant DMS 9500486 .

(C)1999 American Mathematical Society 
Let $A_{r}$ be the $r^{\text {th }}$ Weyl algebra over $k$ with generators $x_{1}, \ldots, x_{r}, \partial_{1}, \ldots, \partial_{r}$ such that $\partial_{i} x_{j}-x_{j} \partial_{i}=\delta_{i j}$.

If $A$ is any $\mathbb{Z}_{2}$-graded associative algebra, we can regard $A$ as a Lie superalgebra by setting

$$
[a, b]=a b-(-1)^{\alpha \beta} b a
$$

where $a, b$ are elements of $A$ of degree $\alpha, \beta$, respectively. We note that $A_{r}$ can be made into a $\mathbb{Z}_{2}$-graded algebra by setting $\operatorname{deg} x_{i}=\operatorname{deg} \partial_{i}=1$.

In [Mo] Montgomery shows that if we consider the $r^{\text {th }}$ Weyl algebra $A_{r}$ as a $\mathbb{Z}_{2}$-graded algebra, then $\left[A_{r}, A_{r}\right] /\left(\left[A_{r}, A_{r}\right] \cap k\right)$ is a simple Lie superalgebra, and that when $r=1, A_{1}=k \oplus\left[A_{1}, A_{1}\right]$.

Using the adjoint representation of $\mathfrak{g}$ on $A_{r}$ we show that $A_{r}=k \oplus\left[A_{r}, A_{r}\right]$ for all $r$. In addition if $r \neq s$, then $\left[A_{r}, A_{r}\right]$ is not isomorphic to $\left[A_{s}, A_{s}\right]$ as a Lie superalgebra. This answers a question of Montgomery.

Much is known about the enveloping algebras of the Lie superalgebras $\operatorname{sp} p(1,2 r)$ $[\mathrm{F}],[\mathrm{M} 1],[\mathrm{M} 2],[\mathrm{P}]$. However, we have tried to keep this paper as self-contained as possible.

Lemma 1. If $\mathfrak{g}$ is a classical simple Lie superalgebra which is not isomorphic to osp $(1,2 r)$ for any $r$, then the only completely prime ideal of $U(\mathfrak{g})$ is the augmentation ideal.

Proof. It is shown in [B, pages 17-20], that if $\mathfrak{g} \neq \operatorname{osp}(1,2 r)$, then $\mathfrak{g}$ contains an odd element $x$ such that $[x, x]=0$. Hence if $P$ is a completely prime ideal, then $x^{2}=0 \in P$ forces $x \in P$. Since $P \cap \mathfrak{g}$ is an ideal of $\mathfrak{g}$, this implies $\mathfrak{g} \subseteq P$.

Lemma 2. If $\mathfrak{g}=\operatorname{osp}(1,2 r)$, there is a surjective homomorphism $U(\mathfrak{g}) \longrightarrow A_{r}$.

Proof. Set

$$
\mathfrak{g}_{1}=\sum_{i} k x_{i}+\sum_{i} k \partial_{i}
$$

and

$$
\mathfrak{g}_{0}=\sum_{i, j} k x_{i} x_{j}+\sum_{i, j} k \partial_{i} \partial_{j}+\sum_{i, j} k\left(x_{i} \partial_{j}+\partial_{j} x_{i}\right) .
$$

We may identify $\mathfrak{g}_{0}$ with the second symmetric power $S^{2} \mathfrak{g}_{1}$ of $\mathfrak{g}_{1}$. Then $\mathfrak{g}=$ $\mathfrak{g}_{0} \oplus \mathfrak{g}_{1}$ becomes a Lie superalgebra under the bracket

$$
[a, b]=a b-(-1)^{\alpha \beta} b a
$$

where $a \in \mathfrak{g}_{\alpha}$ and $b \in \mathfrak{g}_{\beta}$. It follows immediately from the description of $\operatorname{osp}(m, n)$ given in $[\mathrm{K}, 2.1 .2$, supplement $]$ that $\mathfrak{g} \cong \operatorname{osp}(1,2 r)$.

Now let $\mathfrak{a}_{r}$ be the $r^{\text {th }}$ Heisenberg Lie algebra with basis $X_{1}, \ldots, X_{r}, Y_{1}, \ldots$, $Y_{r}, Z$ and nonvanishing brackets given by $\left[X_{i}, Y_{j}\right]=\delta_{i j} Z$. Thus $U\left(\mathfrak{a}_{r}\right) /(Z-1)$ is isomorphic to $A_{r}$ via the map sending $X_{i}$ to $x_{i}$ and $Y_{i}$ to $y_{i}$. By [D, Lemma 4.6.9], $\mathfrak{g}_{0}=s p(2 r)$ acts by derivations on $\mathfrak{a}_{r}$, and hence on $U\left(\mathfrak{a}_{r}\right)$ and on the symmetric algebra $S\left(\mathfrak{a}_{r}\right)$. Therefore by [D, Proposition 2.4.9], the symmetrisation map $w: S\left(\mathfrak{a}_{r}\right) \longrightarrow U\left(\mathfrak{a}_{r}\right)$ is an isomorphism of $\mathfrak{g}_{0}$-modules. Set $S=S\left(\mathfrak{a}_{r}\right) /(Z-1)$. Clearly $w$ induces an isomorphism $\bar{w}: S \longrightarrow A_{r}$ of $\mathfrak{g}_{0}$-modules. Now $S$ is a polynomial algebra in $2 r$ variables, and we let $S(n)$ be the subspace of homogeneous polynomials of degree $n$. Clearly $S(n)$ is a $\mathfrak{g}_{0}$-module.Set $A(n)=\bar{w}(S(n))$. Our 
main result is the following:

Theorem 3. Under the adjoint action

1) $A(n)$ is a simple $\mathfrak{g}_{0}$-module for all $n$.

2) $A(2 n) \oplus A(2 n-1)$ is a simple $\mathfrak{g}$-module for all $n$.

Remark. When $r=1$ the decomposition of the adjoint representation is given in [P, Lemma 7.4.1]. Part 1) of the theorem also follows from arguments in [PS, Section $3]$.

In order to prove the theorem, we need some notation.

For $1 \leq i \leq r-1$, consider the elements of $\mathfrak{g}$ given by

$$
e_{i}=x_{i+1} \partial_{i}, \quad f_{i}=x_{i} \partial_{i+1}
$$

and

$$
h_{i}=\left[e_{i}, f_{i}\right]=x_{i+1} \partial_{i+1}-x_{i} \partial_{i} .
$$

In addition, set $e_{r}=\partial_{r}, f_{r}=x_{r}$ and $h_{r}=-\left[e_{r}, f_{r}\right] / 2=-\left(x_{r} \partial_{r}+\partial_{r} x_{r}\right) / 2$. Then $\mathfrak{h}=\operatorname{span}\left\{h_{i} \mid 1 \leq i \leq r\right\}$ is a Cartan subalgebra of $\mathfrak{g}$. We let $\alpha_{1}, \ldots, \alpha_{r} \in \mathfrak{h}^{*}$ be the positive roots determined by $\left[h, e_{i}\right]=\alpha_{i}(h) e_{i}$ for all $h \in \mathfrak{h}$. The values $\alpha_{i}\left(h_{j}\right)$ are the entries in the (symmetrized) Cartan matrix

$$
\left[\begin{array}{rrrrrr}
2 & -1 & & & & \\
-1 & 2 & -1 & & & \\
& -1 & 2 & \ddots & & \\
& & \ddots & \ddots & -1 & \\
& & & -1 & 2 & -1 \\
& & & & -1 & 1
\end{array}\right] \text {. }
$$

Let $\mathfrak{n}$ be the subalgebra of $\mathfrak{g}$ generated by $e_{1}, \ldots, e_{r}$ and $\mathfrak{n}_{0}=\mathfrak{n} \cap \mathfrak{g}_{0}$. If $L$ is a $\mathfrak{g}$-module (resp. $\mathfrak{g}_{0}$-module), we say that $v \in L$ is a highest weight vector for $\mathfrak{g}$ (resp. for $\mathfrak{g}_{0}$ ) of weight $\lambda \in \mathfrak{h}^{*}$ if $h v=\lambda(h) v$ for all $h \in \mathfrak{h}$ and $\mathfrak{n} v=0$ (resp. $\left.\mathfrak{n}_{0} v=0\right)$.

The bilinear form $($,$) defined on \mathfrak{h}^{*}$ by $\left(\alpha_{i}, \alpha_{j}\right)=\alpha_{i}\left(h_{j}\right)$ is invariant under the action of the Weyl group. For later computations involving $($,$) it is convenient$ to use the following alternative description [K, 2.5.4]. Identify $\mathfrak{h}^{*}$ with $k^{r}$ with standard basis $\epsilon_{1}, \ldots, \epsilon_{r}$ and $($,$) with the usual inner product. Then \alpha_{i}=\epsilon_{i}-\epsilon_{i+1}$ for $1 \leq i \leq r-1$ and $\alpha_{r}=\epsilon_{r}$. Let $\rho_{0}$ (resp. $\rho_{1}$ ) denote the half-sum of the positive even (resp. odd) roots of $\mathfrak{g}$ and $\rho=\rho_{0}-\rho_{1}$. Under the identification above we have $\rho_{0}=\sum_{i=1}^{r}(r-i+1) \epsilon_{i}, \rho_{1}=\frac{1}{2} \sum_{i=1}^{r} \epsilon_{i}$ and $\rho=\frac{1}{2} \sum_{i=1}^{r}(2 r-2 i+1) \epsilon_{i}$.

We now return to the homomorphism $\phi: U(\mathfrak{g}) \longrightarrow A_{r}$. Set $J=\operatorname{Ker} \phi$. Note that $R=\mathbb{C}\left[x_{1}, \ldots, x_{r}\right]$ is a simple $A_{r}$-module and hence a faithful simple $U(\mathfrak{g}) / J$ module. Also $1 \in R$ is a highest weight vector of weight $\lambda$ where $\lambda\left(h_{i}\right)=0$ for $1 \leq i \leq r-1$, and $\lambda\left(h_{r}\right)=-1 / 2$. An easy computation shows that $\lambda=$ $-\frac{1}{2} \sum_{i=1}^{r} i \alpha_{i}=-\rho_{1}$. Thus we have shown

Corollary 4. $J$ is the annihilator of the simple highest weight module with weight $-\rho_{1}$.

We comment briefly on the geometric significance of the embedding $U\left(\mathfrak{g}_{0}\right) / J_{0} \hookrightarrow$ $U(\mathfrak{g}) / J \cong A_{r}$. Passing to the associated graded rings we have

$$
R\left(\bar{O}_{\min }\right)=\operatorname{gr}\left(U\left(\mathfrak{g}_{0}\right) / J_{0}\right) \hookrightarrow \operatorname{gr}\left(A_{r}\right)=R\left(k^{2 r}\right)
$$


where $R(X)$ denotes the ring of regular functions on $X$ and $\bar{O}_{\min }$ is the minimal coadjoint orbit. Let $\mu: k^{2 r} \longrightarrow \mathfrak{g}_{0}^{*}$ be the moment map for the natural action of $S p(2 r)$ on $k^{2 r}$ [CG, 1.4]. Then the image of $\mu$ is contained in $\bar{O}_{\min }$ and the above inclusion is the comorphism $\mu^{*}$. All of this is quite well known. The new twist that Lie superalgebras bring to this situation is a consequence of the next result.

Lemma 5. Suppose $k=\mathbb{C}$, let $\mathfrak{g}=\mathfrak{g}_{0} \oplus \mathfrak{g}_{1}$ be any Lie superalgebra over $\mathbb{C}$ and define $\pi: \mathfrak{g}_{1} \longrightarrow \mathfrak{g}_{0}$ by $\pi(y)=y^{2}\left(=\frac{1}{2}[y, y]\right)$. Then

$$
(\exp \operatorname{ad} x)(\pi(y))=\pi(\exp \operatorname{ad} x(y))
$$

for all $x \in \mathfrak{g}_{0}$ and $y \in \mathfrak{g}_{1}$.

In the above situation, we can identify $\mathfrak{g}_{0}^{*}$ with $\mathfrak{g}_{0}$ and $\mathbb{C}^{2 r}$ with $\mathfrak{g}_{1}$ in such a way that $\mu=\pi$.

Proof. The second claim follows from the first and the uniqueness of the moment map. The formula involving $\pi$ is proved by formally expanding both sides. Define $[x, y]_{0}=y$ and $[x, y]_{n}=(\operatorname{ad} x)^{n}(y)=\left[x,[x, y]_{n-1}\right]$ for $n>0$. Similarly we define $\left[x, y^{2}\right]_{n}$. Then $(\exp \operatorname{ad} x) \pi(y)=\sum_{n \geq 0}\left[x, y^{2}\right]_{n} / n$ !. To show that this equals $\pi(\exp \operatorname{ad} x(y))$ we use the identity

$$
\begin{aligned}
{\left[x, y^{2}\right]_{2 m} } & =\left(\begin{array}{c}
2 m \\
m
\end{array}\right)[x, y]_{m}^{2} \\
& +\sum_{j=0}^{m-1}\left(\begin{array}{c}
2 m \\
j
\end{array}\right)\left[[x, y]_{j},[x, y]_{2 m-j}\right]
\end{aligned}
$$

and a similar identity for $\left[x, y^{2}\right]_{2 m+1}$. The identities are easily proved by induction.

Lemma 6. Under the adjoint action of $\mathfrak{g}_{0}$ or $\mathfrak{g}$ on $A_{r}$,

1) $\partial_{1}^{n}$ is a highest weight vector for $\mathfrak{g}_{0}$ of weight $n \epsilon_{1}$.

2) If $n$ is even, $\partial_{1}^{n}$ is a highest weight vector for $\mathfrak{g}$.

Proof. A simple computation.

If $\lambda \in \mathfrak{h}^{*}$, we denote the simple $\mathfrak{g}_{0}$-module with highest weight $\lambda$ by $L(\lambda)$.

Lemma 7. We have $\operatorname{dim} L\left(n \epsilon_{1}\right)=\left(\begin{array}{c}2 r+n-1 \\ n\end{array}\right)$ for all $n$.

Proof. By Weyl's dimension formula

$$
\operatorname{dim} L(\lambda)=\Pi_{\alpha>0} \frac{\left(\lambda+\rho_{0}, \alpha\right)}{\left(\rho_{0}, \alpha\right)}
$$

where the product is taken over all positive even roots $\alpha$. The even roots $\alpha$ for which $\left(\epsilon_{1}, \alpha\right)>0$ are listed in the first column of the table below. The other columns give the information we need.

\begin{tabular}{|c|c|c|}
\hline$\alpha$ & $\left(\rho_{0}, \alpha\right)$ & $\left(n \epsilon_{1}, \alpha\right)$ \\
\hline$\epsilon_{1}-\epsilon_{i+1}, 1 \leq i \leq r-1$ & $i$ & $n$ \\
\hline$\epsilon_{1}+\epsilon_{j}, 2 \leq j \leq r$ & $2 r-j+1$ & $n$ \\
\hline $2 \epsilon_{1}$ & $2 r$ & $2 n$ \\
\hline
\end{tabular}


Therefore

$$
\operatorname{dim} L\left(n \epsilon_{1}\right)=\prod_{i=1}^{r} \frac{n+i}{i} \prod_{j=2}^{r} \frac{2 r+n-j+1}{2 r-j+1}=\left(\begin{array}{c}
2 r+n-1 \\
n
\end{array}\right) .
$$

Proof of Theorem 3. Set $A=A_{r}$. Part 1) of the theorem follows from Lemmas 6 and 7 , since $\operatorname{dim} A(n)=\left(\begin{array}{c}2 r+n-1 \\ n\end{array}\right)$. Thus $B(n)=A(2 n) \oplus A(2 n-1)$ is a direct sum of two nonisomorphic simple $\mathfrak{g}_{0}$-modules. Also the highest weight vectors $\partial_{1}^{2 n}$ and $\partial_{1}^{2 n-1}$ for these $\mathfrak{g}_{0}$-modules satisfy

$$
\begin{gathered}
{\left[x_{1}, \partial_{1}^{2 n}\right]=-2 n \partial_{1}^{2 n-1},} \\
{\left[\partial_{1}, \partial_{1}^{2 n-1}\right]=2 \partial_{1}^{2 n} .}
\end{gathered}
$$

Let $M$ be the adg-submodule of $A$ generated by $\partial_{1}^{2 n}$. It follows that $B(n) \subseteq M$. Also $M$ is a finite dimensional image of a Verma module (which has a unique simple quotient). On the other hand all finite dimensional simple $\mathfrak{g}$-modules are completely reducible by $[\mathrm{DH}]$. It follows that $M$ is a simple adg-module. (cf. the argument in [Jan, Lemma 5.14]).

We do not know yet that $B(n)$ is an adg-module. This can be seen as follows. We define a filtration $\left\{B_{n}\right\}$ on $A$ by setting $B_{n}=\bigoplus_{m<n} B(m)$. Note that this filtration is the image of the filtration $\left\{U_{n}\right\}$ of $U(\mathfrak{g})$ defined by $U_{n}=U_{1}^{n}$ where $U_{1}=k \oplus \mathfrak{g}$. Hence the associated graded ring $\bigoplus_{n \geq 0} B_{n} / B_{n-1}$ is supercommutative. It follows that $\left[\mathfrak{g}, B_{n}\right] \subseteq B_{n}$ and so $M \subseteq B_{n}$. If $M$ strictly contains $B(n)$, we would have $M \cap(B(n-1) \oplus \ldots \oplus B(1) \oplus k) \neq 0$. By induction, the $B(i)$ with $i<n$ are simple adg-modules, so $M$ would contain $\partial_{1}^{2 i}$ for some $i<n$. However a simple $U(\mathfrak{g})$ module cannot contain more than one highest weight vector. This contradiction shows that $M=B(n)$ and completes the proof.

Theorem 8. We have $\left[A_{r}, A_{r}\right]=\bigoplus_{n>0} A(n)$. In particular $A_{r}=k \oplus\left[A_{r}, A_{r}\right]$.

Proof. Note that if $a, b, c \in A$ have degrees $\alpha, \beta$ and $\gamma$, then as noted in [Mo, Lemma $1.4(3)]$

$$
[a b, c]=[a, b c]+(-1)^{\alpha(\beta+\gamma)}[b, c a] .
$$

Therefore, since $A_{r}$ is generated by the image of $\mathfrak{g}$, we have $\left[A_{r}, A_{r}\right]=\left[A_{r}, \mathfrak{g}\right]$. The result now follows from Theorem 3 .

Remark. From [Mo, Theorem 4.1] it follows that $\left[A_{r}, A_{r}\right]$ is a simple Lie superalgebra for all $r$.

A question raised in $[\mathrm{Mo}]$ is whether, for different $r$, the $\left[A_{r}, A_{r}\right]$ are all nonisomorphic. We show that this is the case by finding the largest rank of a finite dimensional simple Lie subalgebra of $\left[A_{r}, A_{r}\right]$. Note that $s p(2 r) \cong A(2) \subseteq\left[A_{r}, A_{r}\right]$. On the other hand we have

Lemma 9. If $L$ is a finite dimensional simple Lie subalgebra of $\left[A_{r}, A_{r}\right]$, then $\operatorname{rank}(L) \leq r$.

Proof. Note that under the stated hypothesis, $L$ is a Lie subalgebra of $A_{r}$ with the usual Lie bracket $[a, b]=a b-b a$. Now in [J1], Joseph investigates for each simple Lie algebra $L$, the least integer $n=n_{A}(L)$ such that $L$ is isomorphic to a Lie subalgebra of $A_{n}$. (The integer $n_{A}(L)$ is determined to within one for all 
classical Lie algebras.) In particular it follows from Lemma 3.1 and Table 1 of [J1] that $n_{A}(L) \geq \operatorname{rank}(L)$.

Corollary 10. If $\left[A_{r}, A_{r}\right] \cong\left[A_{s}, A_{s}\right]$ as Lie superalgebras, then $r=s$.

For the sake of completeness, we give a proof of Corollary 10 which is independent of [J1]. It is enough to show that if $\mathfrak{g}_{0}=s p(2 r)$ is a Lie subalgebra of a Weyl algebra $A_{n}$, then $n \geq r$. The elements $x_{1} x_{i}, x_{1} \partial_{i}$, with $2 \leq i \leq r$ and $x_{1}^{2}$ span a Heisenberg subalgebra $\mathfrak{a}=\mathfrak{a}_{r-1}$ of $\mathfrak{g}_{0}$ with center spanned by $x_{1}^{2}$. The inclusion $\mathfrak{g}_{0} \subseteq A_{n}$ induces a homomorphism $\phi: U\left(\mathfrak{g}_{0}\right) \longrightarrow A_{n}$. If $I=\operatorname{ker} \phi \cap U(\mathfrak{a})=0$, then we have $G K(U(\mathfrak{a}))=2 r-1 \leq G K\left(A_{n}\right)=2 n$, where $G K()$ denotes Gel'fand-Kirillov dimension, and so $r \leq n$. However if $I \neq 0$, then since the localization of $U(\mathfrak{a})$ at the nonzero elements of $k\left[x_{1}^{2}\right]$ is a simple ring, we would have $x_{1}^{2}-\alpha \in I$ for some scalar $\alpha$. This would imply that $x_{1}^{2}$ is central in $\mathfrak{g}_{0}$, a contradiction.

Remark. It is shown in [Mo, Proposition 4.2] that if $A_{r}$ is isomorphic to $A_{s}$, then $r=s$. Corollary 10 also follows from this and Theorem 8 .

Finally, we note that the proof of Theorem 8 works for certain other algebras.

Theorem 11. Let $\mathfrak{g}$ be a semisimple Lie algebra, and $A$ a primitive factor algebra of $U(\mathfrak{g})$, then $A=k \oplus[A, A]$.

Proof. As before we have $[A, A]=[A, \mathfrak{g}]$. Also $A=\bigoplus V$, a direct sum of finite dimensional simple submodules under the adjoint representation. Since $[V, \mathfrak{g}]$ is a submodule of $V$ for any such $V$, and the center of $A$ equals $k$, we obtain $[A, A]=$ $\bigoplus_{V \neq k} V$, and the result follows.

\section{REFERENCES}

[B] E. Behr, Enveloping algebras of Lie superalgebras, Pacific J. Math 130 (1987), 9-25. MR 89b: 17023

[BFM] Yu. Bahturin, D. Fischman and S. Montgomery, On the generalized Lie structure of associative algebras, Israel J. Math., 96 (1996), 27-48. MR 98d:16048

[CG] N. Chriss and V. Ginzburg, Representation Theory and Complex Geometry, Birkhäuser, Boston 1997. CMP 9:08

[CM] D. H. Collingwood and W. M. McGovern, Nilpotent orbits in semisimple Lie algebras, Van Nostrand Reinhold, New York, 1993. MR 94j:17001

[D] J. Dixmier, Enveloping Algebras, North Holland, Amsterdam 1977. MR 58:16803b

$[\mathrm{DH}]$ D. Z̆. Djoković and G. Hochschild, Semisimplicity of 2-graded Lie algebras, II, Illinois J. Math., 20 (1976) 134-143. MR 52:8206

[F] C. Fronsdal (editor), Essays on Supersymmetry, Mathematical Physics Studies 8, Reidel Publ. Comp., Dordrecht, 1986. MR 88a:81004

$[\mathrm{H}] \quad$ J. E. Humphreys, Introduction to Lie Algebras and Representation Theory, Graduate Texts in Mathematics 9, Springer-Verlag, New York, 1972. MR 48:2197

[He] I. N. Herstein, On the Lie and Jordan rings of a simple associative ring, Amer. J. Math. 77 (1955), 279-285. MR 16:789e

[Jan] J. C. Jantzen, Lectures on Quantum Groups, American Math. Society, 1996. MR 96m:17029

[J1] A. Joseph, Minimal realizations and spectrum generating algebras, Comm. Math. Phys. 36 (1974), 325-338. MR 49:6795

[J2] A. Joseph, The minimal orbit in a simple Lie algebra and its associated maximal ideal, Ann. scient. Éc. Norm. Sup. 9 (1976), 1-29. MR 53:8168

[K] V. Kac, Lie Superalgebras, Adv. in Math. 16 (1977), 8-96. MR 58:5803

[Mo] S. Montgomery, Constructing simple Lie superalgebras from associative graded algebras, J. of Algebra, 195 (1997) 558-579. CMP 98:01 
[M1] I. M. Musson, On the center of the enveloping algebra of a classical simple Lie superalgebra, J. Algebra, 193 (1997), 75-101. CMP 97:14

[M2] I. M. Musson, The enveloping algebra of the Lie superalgebra, osp $(1,2 r)$, Representation Theory, an electronic journal of the AMS, 1 (1997), 405-423. CMP 98:04

[P] G. Pinczon, The enveloping algebra of the Lie superalgebra osp(1,2), J. Alg. 132 (1990), 219-242. MR 91j: 17014

[PS] G. Pinczon and J. Simon, Nonlinear representations of inhomogeneous groups, Lett. Math. Phys. 2 (1978), 499-504. MR 80a:22018

[Sch] M. Scheunert, The theory of Lie superalgebras. An introduction, Lecture Notes in Mathematics, 716, Springer-Verlag, Berlin, 1979. MR 80i:17005

Department of Mathematical Sciences, University of Wisconsin-Milwaukee, MilwauKEE, WISCONSIN 53201

E-mail address: musson@csd.uwm.edu

$U R L$ : www . uwm.edu/ musson/ 